\title{
Pumping of liquids with traveling-wave electroosmosis
}

\author{
A. Ramos ${ }^{\text {a) }}$ \\ Departamento Electrónica y Electromagnetismo, University of Seville, Avenida Reina Mercedes $s / n$. \\ 41012 Sevilla, Spain
}
H. Morgan and N. G. Green
School of Electronics and Computer Science, University of Southampton, Southampton SO17 1BJ, United Kingdom

\begin{abstract}
A. González
Departamento Física Aplicada, Escuela Superior de Ingenieros (E.S.I), University of Seville, Camino de los Descubrimientos s/n. 41092 Sevilla, Spain
\end{abstract}

\begin{abstract}
A. Castellanos
Departamento Electrónica y Electromagnetismo, University of Seville, Avenida Reina Mercedes s/n. 41012 Sevilla, Spain
\end{abstract}

(Received 22 September 2004; accepted 27 January 2005; published online 4 April 2005)

\begin{abstract}
Net flow of electrolyte induced by a traveling-wave electric potential applied to an array of microelectrodes is reported. Two fluid flow regimes have been observed: at small-voltage amplitudes the fluid flow follows the direction of the traveling wave, and at higher-voltage amplitudes the fluid flow is reversed. In both cases, the flow seems to be driven at the level of the electrodes. The experiments have been analyzed with a linear electroosmotic model based upon the Debye-Huckel theory of the double layer. The electrical problem for the experimental interdigitated electrode array is solved numerically using a truncated Fourier series. The observations at low voltages are in qualitative accordance with the electroosmotic model. (C) 2005 American Institute of
\end{abstract} Physics. [DOI: 10.1063/1.1873034]

\section{INTRODUCTION}

The precise control of small masses of liquids is an important requirement in the development of "lab-on-a-chip" devices or micrototal analysis systems ( $\mu$ TAS). ${ }^{1,2}$ Many techniques have been developed to pump liquids in microdevices, including micromechanical methods, ${ }^{3}$ electroosmosis, ${ }^{4}$ electrowetting, ${ }^{5}$ thermocapillary pumping, ${ }^{6}$ electrohydrodynamic (EHD) pumping, ${ }^{7}$ and ac electroosmosis. ${ }^{8}$ In the latter, electrolytic solutions are pumped using asymmetric pairs of microelectrodes subjected to a low potential ac signal of the order of kilohertz. The physical mechanism that causes the electrolyte to move in ac electroosmosis is as follows. If the ac electric field is nonuniform, the component of the ac field normal to the electrode surface induces charge in the double layer, while the tangential component produces a body force on the induced charge, thus moving the fluid. ${ }^{9-14}$ The generated velocity field has a steady-state component which is observed experimentally. Each asymmetric pair of electrodes produces a nonsymmetric local flow that eventually generates a global flow in the direction of broken symmetry. $8,10,15$ Instead of using an asymmetric array and a single-phase ac signal, it is possible to use a traveling-wave signal applied to a symmetric array of electrodes. ${ }^{10,16,17}$ Contrary to EHD pumping, ${ }^{7}$ which also employs a traveling-wave signal, traveling-wave electroosmotic (TWEO) pumping occurs because of electrical forces on the induced charge in the diffuse double layer, while EHD pumping occurs because of electri-

\footnotetext{
a) Author to whom correspondence should be addressed; electronic mail:ramos@us.es
}

cal forces on induced charges that appear in the liquid bulk due to gradients of conductivity and permittivity. ${ }^{18,19}$ In this paper we present some experimental observations of fluid pumping above an electrode array subjected to a travelingwave signal, and a theoretical, linear study of the TWEO pumping mechanism for such array.

The traveling-wave electrode array is also used to induce dielectrophoretic motion of particles (traveling-wave dielectrophoresis. ${ }^{20-23}$ ) The mechanism responsible for particle motion can therefore on occasion be ambiguous. The analysis presented in this paper will aid in discriminating between particle motion induced by dielectrophoresis and particle motion due to fluid flow in traveling-wavedielectrophoretic experiments.

The mechanism responsible for the fluid pumping at low voltages is as follows. Above the electrode array there is an electrolyte, such as $\mathrm{KCl}$, with conductivity $\sigma$ and permittivity $\varepsilon$. At the interfaces between the metal and electrolyte, and glass and electrolyte, double layers are formed. The characteristic thickness of a double layer is given by the Debye length, $\lambda_{D}$, which is much smaller than typical electrode dimensions. A traveling-wave potential is applied to the electrodes as shown in Fig. 1. Ions accumulate in the double layer in response to the applied signal. Due to the finite time during which the double layer is charged, a delay exists between the time of maximum-induced charge and the maximum applied signal. The electric field acts on the charge pulling the fluid in the direction of the traveling wave. The charging of the double layer is analogous to the charging of a capacitor (the double layer) through a resistor (the ohmic 


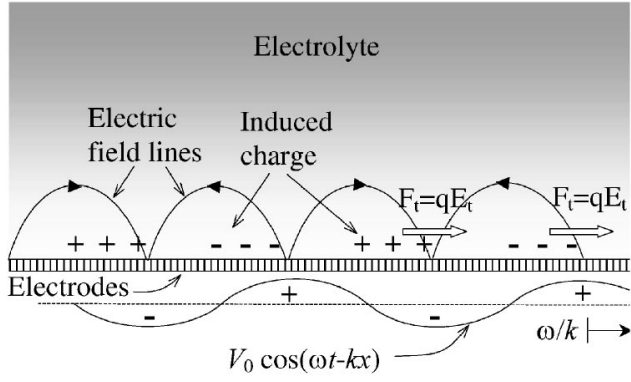

Traveling wave applied potential

FIG. 1. Diagram illustrating the physical mechanism of traveling-wave electroosmosis

liquid). ${ }^{9,11-13}$ The charging time is therefore of order of $\tau_{c}$ $=(\varepsilon / \sigma)\left(\ell / \lambda_{D}\right)$, where $\ell$ is a typical length of the system and $\varepsilon / \lambda_{D}$ is the surface capacitance of the double layer. When the frequency of the applied signal is low $\omega \tau_{c} \ll 1$, most of the applied voltage is dropped across the double layer, and the tangential electric field is small. Conversely, when the frequency is high $\omega \tau_{c} \gg 1$, most of the applied voltage is dropped across the bulk electrolyte and the charge induced in the double layer is small. The frequency of maximum velocity is, therefore, of the order of $\omega_{c} \sim 1 / \tau_{c}$, which is several orders of magnitude smaller than the charge relaxation frequency $\sigma / \varepsilon$. Fluid flow due to ac electroosmosis is observed in the region of this characteristic frequency $\omega_{c} .{ }^{8,9,11,13}$

Ehrlich and Melcher studied theoretically the fluid motion induced by traveling wave in a semi-insulating bipolar liquid. ${ }^{16}$ As in the present work, they took into account the charge induced in the double layer. For small field excitations, they obtained as the characteristic frequency of maximum velocity $\omega \sim \sigma / \varepsilon$. However, they analyzed a system with a thick dielectric layer between the electrodes and the liquid, which is different from the system presented in this paper where the electrodes and the electrolyte are in contact. In the process of finalizing this manuscript, a paper on electroosmotic flows driven by traveling electric fields has appeared. ${ }^{17}$ These authors studied a traveling-wave device with a dielectric layer between the electrodes and the electrolyte, and obtained experimental frequencies of the order of $\sigma / \varepsilon$, as in the case studied by Ehrlich and Melcher. Their theoretical, linear analysis employs a single Fourier mode to describe the electrical potential at any frequency. Here we will present a Fourier series analysis in order to deal with the electrical boundary conditions, that is valid at frequencies $\omega \ll \sigma / \varepsilon$.

In this paper we present experimental observations of unidirectional fluid flow due to the traveling electric field. We then describe a theoretical, linear analysis of travelingwave electroosmosis and compare this theory with the experimental results.

\section{EXPERIMENTAL OBSERVATION OF FLUID MOTION}

Observations of fluid motion were made on an array of $N=20$ interdigitated microelectrodes fabricated on a planar glass slide using photolithography. The microelectrodes were made from a metal trilayer of $10-\mathrm{nm}$ titanium, $100-\mathrm{nm}$ gold, and 20-nm titanium. The purpose of the top layer of titanium

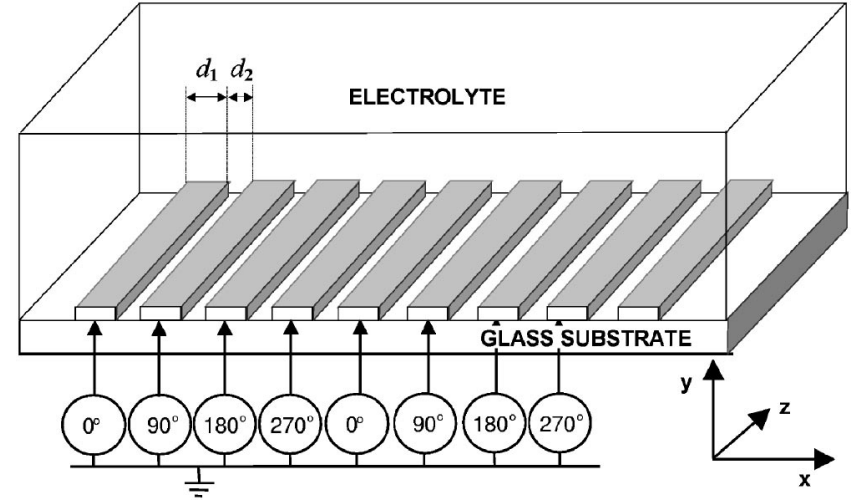

FIG. 2. Diagram showing the experimental arrangement of an electrode array used for traveling-wave electroosmosis.

was to reduce the effects of corrosion at low frequencies. The array consisted of $20-\mu \mathrm{m}$-wide electrodes separated by $20-\mu \mathrm{m}$ gaps. A diagram of the system used to generate the fluid flow is shown in Fig. 2.

The electrodes were connected to an ac voltage of angular frequency $\omega$ and amplitude $V_{0}$, i.e., $V(t)=V_{0} \cos \omega t$. The voltage on consecutive electrodes was phase shifted by $90^{\circ}$, so that the traveling wave had a wavelength $L=160 \mu \mathrm{m}$. A square glass chamber was constructed around the electrode array and filled with electrolyte. The electrode/electrolyte system could be observed both from above and from the side. A microscope objective and camera were pointed horizontally along the electrodes, so that the electrodes could be imaged in cross section. ${ }^{13}$ A solution of $\mathrm{KCl}$ in water of conductivity $2 \mathrm{mS} / \mathrm{m}$ was used for the electrolytic solution. The voltage amplitude $V_{0}$ ranged from 0 to $3 \mathrm{~V}$, and the frequency $f=\omega / 2 \pi$ from $100 \mathrm{~Hz}$ to $100 \mathrm{kHz}$. Electrolysis was not observed at the electrodes because the capping layer of titanium forms a very thin insulating oxide layer. Fluorescent latex particles of $557 \mathrm{~nm}$ in diameter were used as tracers to observe the fluid flow. The movement of the particles was recorded on video and transferred to a computer.

After applying the traveling-wave voltage to the microelectrode array, the fluid moved across the electrode array as shown by the composite image of particle tracks in Fig. 3. It was observed that the fluid flow was driven at the electrode

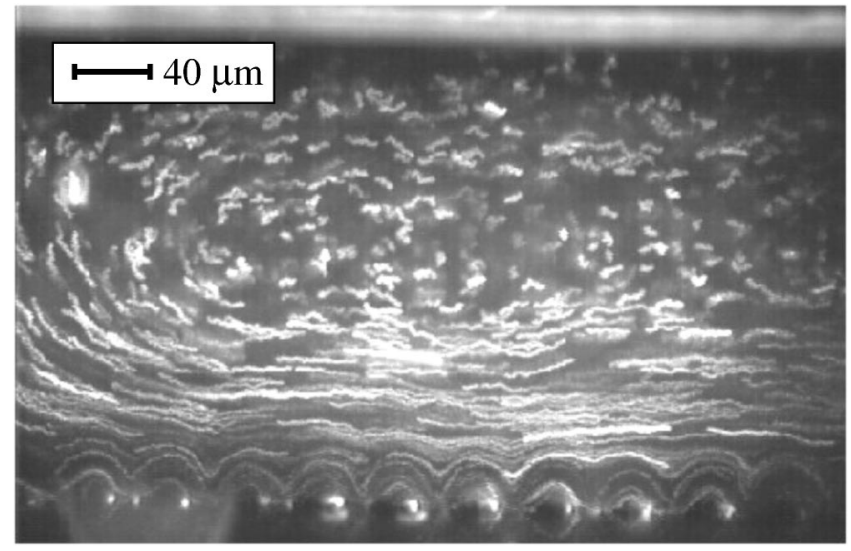

FIG. 3. Superimposed successive video frames showing the experimental streamlines for the case $V_{0}=1.2 \mathrm{~V}$ and $f=1 \mathrm{kHz}$. 


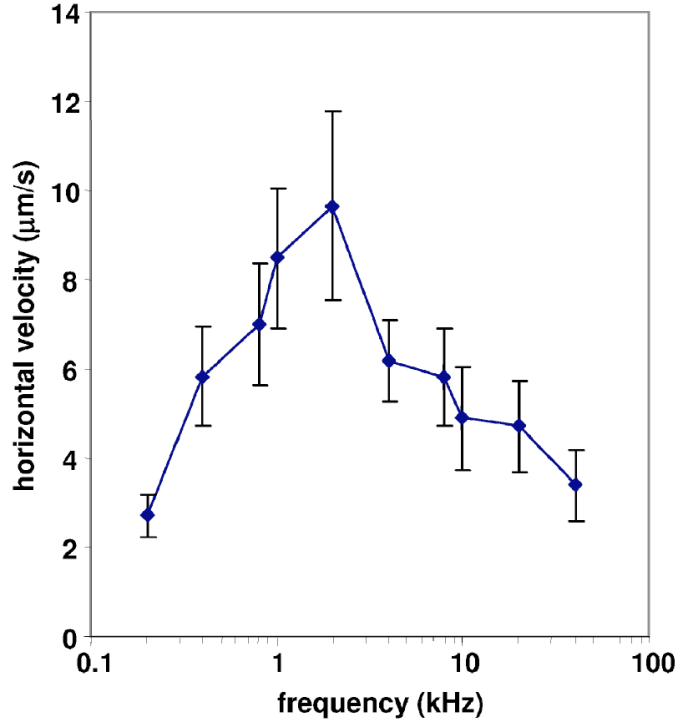

FIG. 4. Measured velocity as a function of frequency for $V_{0}=1 \mathrm{~V}$ and at a height of $65 \mu \mathrm{m}$ from the electrodes.

surface, with highest velocities observed close to the electrode. For $V_{0}<2 \mathrm{~V}$, a net flow was observed which was in the direction of the traveling wave, to the left in Fig. 3, as expected by the qualitative picture of TWEO described in the introduction. Because the chamber is closed at the right and left ends, the fluid recirculates, going from left to right at the top. Some small flow recirculation was also observed at the front and rear walls. In order to determine if the particle movement was indeed due to fluid flow, the expected dielectrophoretic velocity was calculated. For particles of $557 \mathrm{~nm}$ in diameter in this array, the horizontal dielectrophoretic velocity was estimated to be less than $0.5 \mu \mathrm{m} / \mathrm{s}$ at $V_{0}=2 \mathrm{~V}$ and at heights greater than $20 \mu \mathrm{m} .^{21,22}$ Therefore, the effect of dielectrophoresis on particle behavior can be neglected, except very close to the electrode edges, where positive dielectrophoresis can become important.

For the images shown in Fig. 3, the frequency and voltage amplitude of the applied signal were $1 \mathrm{kHz}$ and $1.2 \mathrm{~V}$, respectively. At a height of $30 \mu \mathrm{m}$ from the surface, velocities of approximately $30 \mu \mathrm{m} / \mathrm{s}$ were observed. Latex particles were seen to accumulate at the left edge of each electrode, probably due to dielectrophoresis.

Figure 4 is a plot of the fluid velocity for different applied frequencies, for a height of approximately $64 \mu \mathrm{m}$, and a voltage amplitude of $1 \mathrm{~V}$. The figure shows that the fluid flow occurs in a frequency window centered at approximately $2 \mathrm{kHz}$. As expected from ac electroosmosis, this frequency is much smaller than the charge relaxation frequency $\sigma / 2 \pi \varepsilon(\simeq 0.5 \mathrm{MHz}$ for the conductivity used in the experiments). The characteristic frequency of ac electroosmosis $f_{c}=\omega_{c} / 2 \pi \sim 680 \mathrm{~Hz}(\ell=20 \mu \mathrm{m})$ is of the order of the observed frequency $2 \mathrm{kHz}$ for maximum velocity.

The fluid velocity was also measured at three different heights $(29,42$, and $54 \mu \mathrm{m})$ as a function of voltage, and this is shown in Fig. 5. The frequency of the applied signal was $1 \mathrm{kHz}$. The estimated averaged velocity at the level of the electrodes is also shown. This was estimated by assuming the velocity profile to be parabolic (as a function of height), and

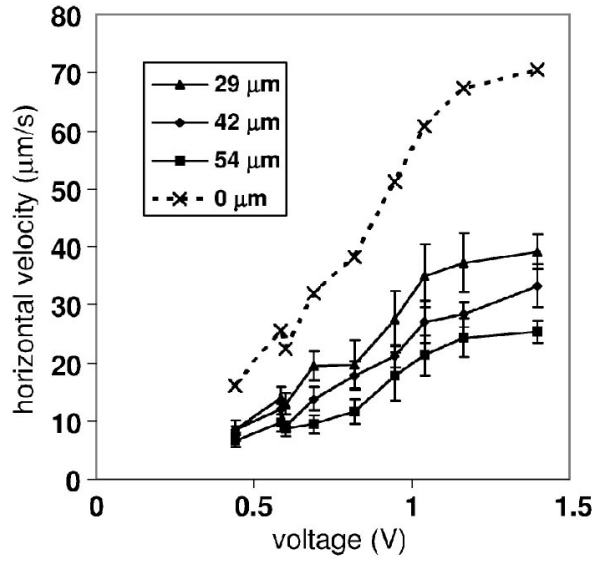

FIG. 5. Measured velocity as a function of voltage at $f=1 \mathrm{kHz}$ for three different heights, and estimated velocity at the level of the electrodes.

extrapolating back to the level of the electrodes $(0 \mu \mathrm{m})$. The measured velocities can be approximated to a parabolic profile by setting the velocity to zero at $h=97 \mu \mathrm{m}$ due to the recirculating flow and at $h=219 \mu \mathrm{m}$ due to the nonslip condition at the upper wall. Theoretically, a parabolic function is obtained from the Stokes equation with no volumetric force and assuming a unidirectional flow. Although there is scatter in the data, this is not significant, and saturation is observed above a voltage amplitude of $V_{0}=1 \mathrm{~V}$.

For voltage amplitudes $V_{0} \geqslant 2 \mathrm{~V}$ and at $1 \mathrm{kHz}$, the fluid flow changed direction, a phenomenon that is not expected from our understanding of ac electroosmosis. The observed recirculation of the tracking particles was an indication of fluid motion. Figure 6 shows the velocity of the fluid as a function of height in the channel at three different voltages for a frequency of $1 \mathrm{kHz}$. It can be seen that the velocity is nearly zero at the midpoint of the channel for the lower voltages $(1.2$ and $1.4 \mathrm{~V})$, but that the change in behavior at $2.4 \mathrm{~V}$ is noticeable, both in terms of the flow direction and the large jump in fluid velocity, particularly close to the electrodes. In this case, the velocities at a height of $20 \mu \mathrm{m}$ above the electrodes were around $150 \mu \mathrm{m} / \mathrm{s}$ for a voltage amplitude of 2.4 $\mathrm{V}$ and frequency of $1 \mathrm{kHz}$. In general, higher voltages were

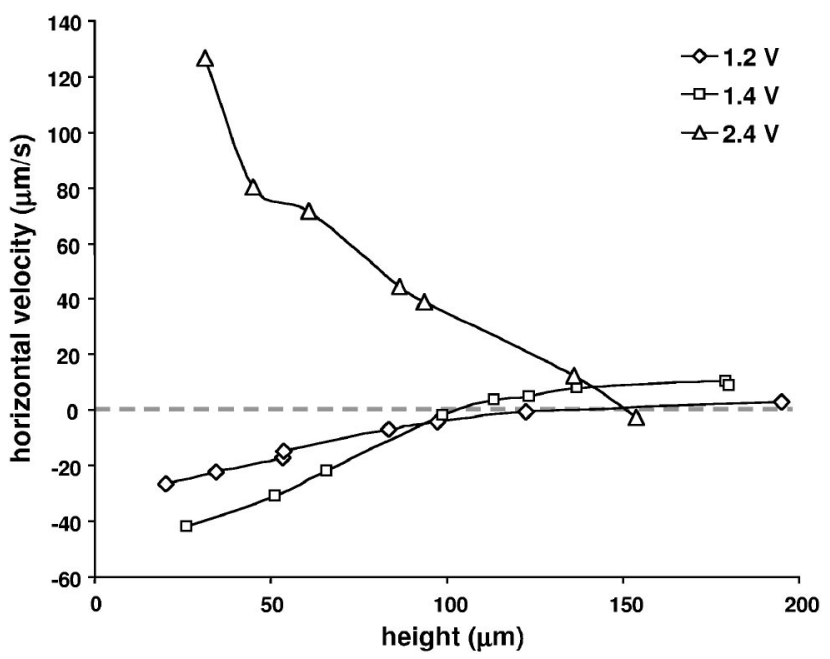

FIG. 6. Measured velocity as a function of height for three different voltage amplitudes and $f=1 \mathrm{kHz}$. 
required in order to observe this kind of reversal flow at higher frequencies. These voltages are too low to ascribe the flow to the electrothermal mechanism for this conductivity. ${ }^{19}$

Reversal of the fluid direction was also observed above a similar threshold voltage when gold electrodes were used. Reversals of fluid flow with increasing voltage have also been observed using arrays of asymmetric pairs of electrodes, ${ }^{24,25}$ which could be related to our observations for traveling-wave arrays. Although the effect is reproducible, the origin of this phenomenon is not clear. The numerical solutions given by Ehrlich and Melcher ${ }^{16}$ showed some cases of reversed pumping, although at angular frequencies of the order of $\sigma / \varepsilon$, much higher than in our experiments. Their numerical and analytical studies always indicated forward pumping at frequencies $\omega \varepsilon / \sigma \ll 1$. At voltages much greater than the thermal voltage $k_{B} T / e$, concentration polarization should become important and lateral concentration gradients could produce diffusio-osmosis. ${ }^{26,27}$ Also, Faradaic charge injection from the electrodes to the electrolyte could be possible without observable electrolysis. Washabaugh et al. ${ }^{28}$ observed forward and backward pumping of semi-insulating liquids pumped by a traveling-wave potential. They assumed that charge injection was responsible for the fluid motion. As the charge is injected, the sign of the charge layer close to the electrodes could change and, therefore, the direction of electrical force and slip velocity. However, their liquids are quite different to ours. For semi-insulating liquids, the applied electric field is usually much greater than the typical electric field in the double layer $k_{B} T / e \lambda_{D}$. For our system this electric field would be around $10^{6} \mathrm{~V} / \mathrm{m}$, greater than typical applied field required for reverse pumping, which is of the order of $10^{5} \mathrm{~V} / \mathrm{m}(2 \mathrm{~V} / 20 \mu \mathrm{m})$. Very recently, fluid flow of electrolyte subjected to ac voltages with velocities of the order of $1 \mathrm{~mm} / \mathrm{s}$ has been reported, ${ }^{29}$ and the mechanism responsible for the fluid flow has been ascribed to Faradaic charging. Although, the electrode geometry is quite different to the traveling-wave array, the underlying mechanism could be the same.

\section{THE LINEAR ELECTROKINETIC PROBLEM}

Linear electrokinetic theory was used to study the system shown in Fig. 2. As shown in the figure, the system is modeled as an insulating substrate (glass) onto which an infinite periodic array of microelectrodes is fabricated. The electrodes are considered to be infinitely long and thin with width $d_{1}$ and spacing $d_{2}$. Four ac signals of the same amplitude $V_{0}$ and angular frequency $\omega$, and relative phases $0^{\circ}, 90^{\circ}$, $180^{\circ}$, and $270^{\circ}$ are applied to the array, so that the voltage applied to consecutive electrodes is phase shifted by $90^{\circ}$. The spatial period of the system is $L=4 d_{1}+4 d_{2}$. A symmetrical electrolyte, such as $\mathrm{KCl}$, is placed on top of the array. In most cases, the Debye length $\lambda_{D}$ is of the order of $10 \mathrm{~nm}$ and is negligibly small compared to the other lengths in the system, which are of the order of $10 \mu \mathrm{m}$. To a first approximation we assume that the voltage applied to the electrodes is low enough such that electrolysis of the electrolyte does not occur. If the frequency of the applied signal is also low enough, i.e., $\omega \ll \sigma / \varepsilon$, the double layer can be assumed to be in quasiequilibrium. ${ }^{30}$ Under these conditions the bulk electrolyte behaves in a resistive manner and the double layer considered to be an ideal capacitor. Therefore, the electrical potential in the bulk electrolyte satisfies Laplace's equation

$$
\nabla^{2} \Phi=0 .
$$

The boundary condition on the electrode surface describes the charging of the double layer due to the current in the bulk, $\partial q_{s} / \partial t=-\sigma E_{y}$, with $q_{s}$ the charge per unit area in the double layer. For sufficiently low voltage across the diffuse layer $\left(\Delta \Phi<k_{B} T / e\right)$, there is a linear relationship between the surface charge and the voltage drop across the double layer. In this case, the surface charge conservation equation can be written using phasors as

$$
\sigma \frac{\partial \Phi}{\partial y}=i \omega C_{\mathrm{DL}}\left(\Phi-V_{j}\right),
$$

where $i=\sqrt{-1}, C_{\mathrm{DL}}$ is the capacitance per unit of area of the whole double layer (diffuse plus compact or Stern layers), $\Phi$ is the potential just outside the double layer, and $V_{j}$ is the potential applied to electrode $j$. At the interface between the electrolyte and the glass a similar boundary condition holds. For frequencies $\omega \ll \sigma / \varepsilon$, this boundary condition can be simplified to ${ }^{13,15}$

$$
\frac{\partial \Phi}{\partial y}=0 \text {. }
$$

In defining both boundary conditions we have implicitly ignored any lateral conduction current along the double layer. Also, any current due to convection in the system has been neglected. The boundary condition at $y \rightarrow \infty$ is the potential tends to zero.

Once the electric potential has been solved, the electroosmotic fluid velocity at the surface of the electrodes can be calculated. For diffuse double layers in quasiequilibrium on perfectly polarizable metal surfaces, the electroosmotic slip velocity is given by the Helmholtz-Smoluchowski formula, ${ }^{13,31}$

$$
u=\frac{\varepsilon \Delta \Phi}{\eta} E_{x} .
$$

Here $\eta$ is the viscosity of the fluid, $\Delta \Phi=\Phi-\Phi_{0}$ is the potential drop across the diffuse double layer $\left(\Phi_{0}\right.$ is the potential at the nonslip plane), and $E_{x}$ is the tangential electric field outside the double layer. For our problem, both $\Delta \Phi$ and $E_{x}$ are oscillating functions of time, of frequency $\omega$. The time-averaged slip fluid velocity at the interface between the double layer and the bulk is ${ }^{12,13}$

$$
\langle u\rangle=-\frac{\varepsilon}{4 \eta} \Lambda \frac{\partial}{\partial x}\left[\left(\Phi-V_{j}\right)\left(\Phi-V_{j}\right)^{*}\right],
$$

where $*$ indicates complex conjugate and $\Lambda$ is the ratio of the diffuse double-layer voltage to the total double-layer voltage. The parameter $\Lambda$ is given by $\Lambda=C_{s} /\left(C_{s}+C_{d}\right)$, where $C_{d}$ and $C_{s}$ are the capacitances of the diffuse and Stern or compact layers, respectively. For the glass/electrolyte interface, an estimate of the potential drop across the diffuse double layer shows that this is negligibly small and, from Eq. (4), the 
electroosmotic velocity on the glass is negligible. ${ }^{15}$

To obtain the bulk velocity, the Navier-Stokes equations are solved in the limit of small Reynolds number, usually very small in microsystems. In the steady state, and in the absence of externally applied body forces, the equations reduce to

$$
\eta \nabla^{2} \mathbf{u}-\nabla p=0, \quad \nabla \mathbf{u}=0
$$

where $p$ is the pressure and $\mathbf{u}$ the velocity, $\mathbf{u}=u \mathbf{e}_{x}+v \mathbf{e}_{y}$. The boundary conditions for these equations at $y=0$ are

(a) the tangential velocity is equal to the slip velocity on the electrodes, given by Eq. (5);

(b) the tangential velocity is zero at the glass;

(c) the normal velocity is zero for any $x$ at $y=0$ (electrodes and glass).

Far from the electrodes in the normal direction, the fluid can be considered to be free of stress, $\partial u / \partial y=0$ and $v=0$. Given the periodicity of the electrode array we look for periodic solutions in the $x$ direction.

The velocity field can be obtained from an integration of the slip velocity calculated from the electrical problem. ${ }^{15}$ Defining the stream function $\Psi$ by the pair of derivatives $\partial \Psi / \partial y=u$ and $\partial \Psi / \partial x=-v$, the function $\Psi$ that is solution of the Stokes problem in the bulk and satisfies the abovementioned boundary conditions can be written as ${ }^{15}$

$$
\Psi=\frac{1}{L} \int_{0}^{L} d x^{\prime} u\left(x^{\prime}, 0\right) \frac{y \sinh \left(k_{0} y\right)}{\cosh \left(k_{0} y\right)-\cos \left[k_{0}\left(x-x^{\prime}\right)\right]},
$$

where $u\left(x^{\prime}, 0\right)$ is the slip velocity calculated from the electrical problem and $k_{0}=2 \pi / L$. If a net fluid flow occurs, the velocity tends to $\mathbf{u}=U \mathbf{e}_{x}$ for $y \rightarrow \infty$, where $U$ is a constant. This condition presumes that the upper boundary of any actual device is very much higher than the wavelength of the problem. The value of the pumping velocity $U$ is equal to the average value of the slip velocity over a wavelength, i.e., $U=(1 / L) \int_{0}^{L} u d x$. This equality can be seen, for instance, from expression (7) taking the leading terms when $k_{0} y \gg 1$.

\section{MATHEMATICAL ANALYSIS}

\section{A. Single potential wave}

We first present the analysis for the case of a single potential wave which is simpler than the potential wave for the four-phase electrode array. If the potential applied at the level of the electrodes is approximated by a single potential wave of angular frequency $\omega$ and wave number $k_{0}=2 \pi / L$, of the form $V_{0} e^{i\left(\omega t-k_{0} x\right)}$, the solution for the electrical potential in the bulk is of the form $\Phi=A e^{-k_{0} y} e^{i\left(\omega t-k_{0} x\right)}$. Applying the charge conservation boundary condition at $y=0$

$$
\sigma \frac{\partial \Phi}{\partial y}=i \omega C_{\mathrm{DL}}\left(\Phi-V_{0} e^{-i k_{0} x}\right)
$$

we obtain

$$
A=\frac{i \Omega V_{0}}{1+i \Omega}
$$

where $\Omega=\omega C_{\mathrm{DL}} /\left(\sigma k_{0}\right)$. The generated electroosmotic slip velocity is

$$
u=-\frac{\varepsilon}{2 \eta} \operatorname{Re}\left[\Delta \Phi \frac{\partial \Phi^{*}}{\partial x}\right]=\Lambda \frac{\varepsilon k_{0} V_{0}^{2}}{2 \eta} \frac{\Omega}{1+\Omega^{2}} .
$$

The velocity $u$ is independent of the coordinate $x$, then the pumping velocity $U=u$. The maximum velocity as a function of frequency is obtained for $\Omega=1$ and is

$$
U=\Lambda \frac{\varepsilon k_{0} V_{0}^{2}}{4 \eta}
$$

Equation (10) is in complete agreement with the velocity expression for low frequencies given in Ref. 17.

Ehrlich and Melcher studied traveling-wave-induced motion in a bipolar semi-insulating liquid theoretically. ${ }^{16}$ Their model described the generation and recombination of bipolar carriers. Unlike our case, their system had a thick dielectric solid, which acted as a conduit wall, between the electrodes and the liquid. They studied the case of a pure traveling wave at different ranges of electric-field amplitude and signal frequencies. For small field amplitudes, they obtained a maximum velocity of $0.2 \varepsilon E_{0}^{2} \lambda_{D} / \eta$ for a frequency $\omega=\sigma / \varepsilon$. Here $E_{0}$ is the normal electric field inside the double layer at the nonslip plane. This frequency of maximum velocity is much higher than for our case, $\omega=\sigma k_{0} / C_{\mathrm{DL}}$ $\sim k_{0} \lambda_{D} \sigma / \varepsilon$. Analyzing their expression of the slip velocity for small field and low frequency $(\omega \ll \sigma / \varepsilon)$

$$
u=\frac{\varepsilon E_{0}^{2} \lambda_{D}}{2 \eta} \frac{\omega \varepsilon}{\sigma}
$$

we can reconcile their results with ours. In this expression, $E_{0}$ is independent of frequency. However, if the electrodes are in contact with the electrolyte (or separated by a very thin insulator, e.g., oxide layer as for Ti electrodes) then the field is a function of frequency. In this case, the charging time of the double layer $\tau_{c}=\varepsilon L / \sigma \lambda_{D}$ becomes crucial, as explained in the Introduction. The voltage across the diffuse double layer and, therefore, the field inside the double layer $E_{0}$ are maximum at $\omega \tau_{c} \ll 1$, and decrease with increasing frequency. Equating $E_{0}^{2}$ to $|\Delta \Phi|^{2} / \lambda_{D}^{2}$ in Eq. (12), and taking into account the frequency dependency of $\Delta \Phi$, we recover the slip velocity expression (10).

Equation (11) indicates that the maximum slip velocity decreases as the thickness of the dielectric layer increases, through the scaling factor $\Lambda$. With a thick dielectric conduit, as in the case studied by Ehrlich and Melcher, the slip velocity becomes inversely proportional to the thickness of the dielectric layer. Therefore, larger voltages are needed to produce fluid velocities of the same order of those obtained working with electrodes in contact with the electrolyte.

\section{B. The traveling-wave array}

The electrical potential for the four-phase traveling-wave electrode array can be solved numerically using Fourier series. The electrical potential can be written as $\Phi=\Phi_{1}+\Phi_{2}$, 

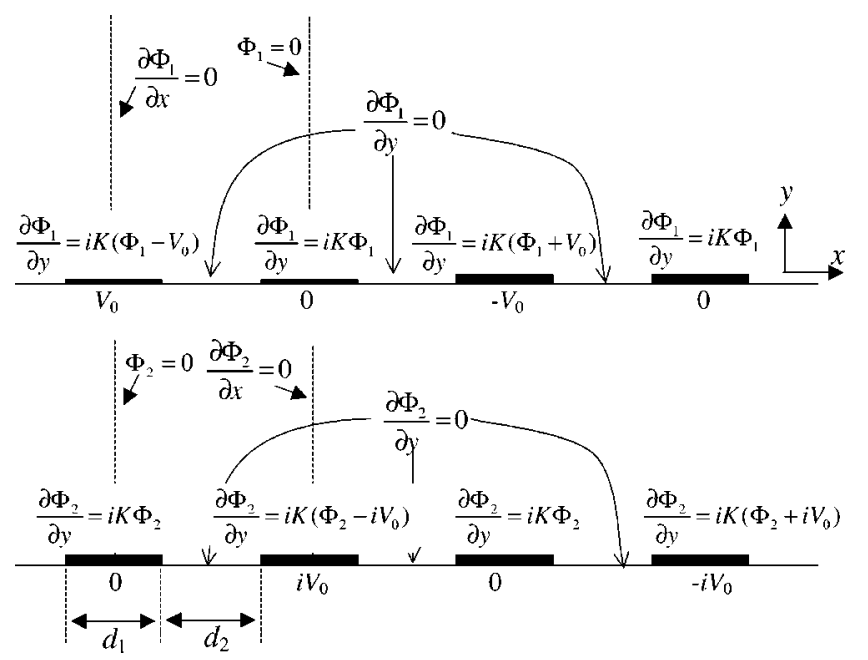

FIG. 7. Boundary conditions for $\Phi_{1}$ and $\Phi_{2}$.

where $\Phi_{1}$ is the solution for a problem with boundary conditions as shown in Fig. 7(a) and $\Phi_{2}$ as shown in Fig. 7(b). Furthermore, the boundary conditions for $\Phi_{2}$ are those for $\Phi_{1}$ shifted a distance $\left(d_{1}+d_{2}\right)$ with $V_{0}$ changed to $-i V_{0}$. Therefore, $\Phi_{2}$ can be written as

$$
\Phi_{2}(x, y)=-i \Phi_{1}\left[x-\left(d_{1}+d_{2}\right), y\right] .
$$

For $\Phi_{1}, x=0$ is a line of even symmetry, i.e., $\partial \Phi_{1} / \partial x$ $=0$, while $x=d_{1}+d_{2}=L / 4$ is a line of odd symmetry, i.e., $\Phi_{1}=0$. Taking these into account, the general form of $\Phi_{1}$ using Fourier series is

$$
\Phi_{1}=\sum_{n=0}^{\infty} A_{n} \cos \left(k_{n} x\right) e^{-k_{n} y}
$$

with $k_{n}=(2 n+1) 2 \pi / L$. The boundary conditions at $y=0$ for $\Phi_{1}$ impose

$$
\begin{gathered}
-\sum_{n=0}^{\infty} k_{n} A_{n} \cos \left(k_{n} x\right)=-i K V_{0}+i K \sum_{n=0}^{\infty} A_{n} \cos \left(k_{n} x\right) \\
0<x<d_{1} / 2, \\
-\sum_{n=0}^{\infty} k_{n} A_{n} \cos \left(k_{n} x\right)=0 \quad d_{1} / 2<x<d_{1} / 2+d_{2}, \\
-\sum_{n=0}^{\infty} k_{n} A_{n} \cos \left(k_{n} x\right)=i K \sum_{n=0}^{\infty} A_{n} \cos \left(k_{n} x\right) \\
d_{1} / 2+d_{2}<x<d_{1}+d_{2},
\end{gathered}
$$

where $K=C_{\mathrm{DL}} \omega / \sigma$. Multiplying the Fourier representation of $\partial \Phi_{1} / \partial y$ by $\cos \left(k_{m} x\right)$ and integrating in the interval from 0 to $d_{1}+d_{2}$ we arrive at

$$
-k_{m} d A_{m}=-i K V_{0} c_{m}+i K \sum_{n=0}^{\infty} A_{n} a_{m n},
$$

where $d=\left(d_{1}+d_{2}\right) / 2$ and

$$
c_{m}=\int_{0}^{d_{1} / 2} \cos \left(k_{m} x\right) d x
$$

$$
a_{m n}=\delta_{m n} d-\int_{d_{1} / 2}^{d_{1} / 2+d_{2}} \cos \left(k_{n} x\right) \cos \left(k_{m} x\right) d x .
$$

We solve the linear system (18) numerically by truncating the series.

The potential of the original problem is

$$
\begin{aligned}
\Phi & =\Phi_{1}(x, y)-i \Phi_{1}\left[x-\left(d_{1}+d_{2}\right), y\right] \\
& =\sum_{n=0}^{\infty} A_{n}\left\{\cos \left(k_{n} x\right)-i \cos \left[k_{n}(x-2 d)\right]\right\} e^{-k_{n} y}, \\
& =\sum_{n=0}^{\infty} A_{n}\left[\cos \left(k_{n} x\right)-i(-1)^{n} \sin \left(k_{n} x\right)\right] e^{-k_{n} y} \\
& =\sum_{n=0}^{\infty} A_{n} e^{-k_{n} y} e^{-i k_{n} x(-1)^{n}} .
\end{aligned}
$$

The potential is the superposition of waves propagating alternately in the positive or negative $x$ direction depending on whether $n$ is even or odd, respectively. From the potential, the electroosmotic slip velocity can be calculated and therefore the average velocity in a wavelength, the pumping velocity $U$. Because of the symmetry the average velocity on each electrode should be equal,

$$
\begin{aligned}
U & =\frac{1}{L} \int_{0}^{L} u d x=\frac{4}{L} \int_{-d_{1} / 2}^{d_{1} / 2} u d x \\
& =\Lambda \frac{\varepsilon}{L \eta}\left(\left|\Phi-V_{0}\right|_{x=-d_{1} / 2}^{2}-\left|\Phi-V_{0}\right|_{x=d_{1} / 2}^{2}\right) .
\end{aligned}
$$

Another expression can be obtained from Eq. (2)

$$
u=-\frac{\varepsilon}{2 \eta} \operatorname{Re}\left[\Delta \Phi \frac{\partial \Phi^{*}}{\partial x}\right]=\Lambda \frac{\varepsilon}{2 \eta} \operatorname{Re}\left[\frac{i \sigma}{\omega C_{\mathrm{DL}}} \frac{\partial \Phi}{\partial y} \frac{\partial \Phi^{*}}{\partial x}\right] \text {. }
$$

This last expression is also valid on the gaps between electrodes, where $\partial \Phi / \partial y=0$ and $u=0$. The pumping velocity is, therefore,

$$
U=\Lambda \frac{\varepsilon}{2 \eta L} \operatorname{Re}\left[\frac{i \sigma}{\omega C_{\mathrm{DL}}} \int_{0}^{L} \frac{\partial \Phi}{\partial y} \frac{\partial \Phi^{*}}{\partial x} d x\right] .
$$

Taking into account the Fourier expression of the potential

$$
U=\Lambda \frac{\varepsilon}{2 \eta} \frac{\sigma}{\omega C_{\mathrm{DL}}} \sum_{n=0}^{\infty}(-1)^{n} k_{n}^{2}\left|A_{n}\right|^{2} .
$$

Sometimes in experiments, the traveling wave is generated using square-wave signals applied to the electrodes instead of sinusoidal signals. ${ }^{7,17}$ We extend the present analysis to traveling-square-wave signals as follows. In order to compute the slip velocity, the voltage applied to the electrodes ( $V_{0}$ in a half period and $-V_{0}$ in the other) should be written as a superposition of sinusoidal waves: electrodes 1 and 3 are subjected to $V_{1}=-V_{3}=\sum_{p=0}^{\infty} B_{p} \cos [(2 p+1) \omega t]$ with $B_{p}$ $=4 V_{0}(-1)^{p} / \pi(2 p+1)$, while electrodes 2 and 4 are subjected to $V_{2}=-V_{4}=\sum_{p=0}^{\infty} B_{p}^{\prime} \sin [(2 p+1) \omega t]$ with $B_{p}^{\prime}=4 V_{0} / \pi(2 p+1)$. As a consequence, the potential in the system is a superposition of potentials with frequencies $\omega, 3 \omega, 5 \omega,(2 p+1) \omega$, 


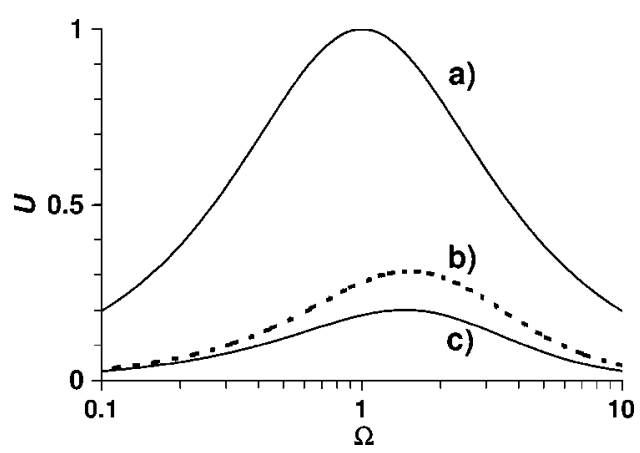

FIG. 8. Pumping velocity $U$ (in units of $\Lambda \varepsilon k_{0} V_{0}^{2} / 4 \eta$ ) vs nondimensional frequency $\Omega$ in TWEO: (a) single mode, (b) square waves applied to electrode array, (c) and sinusoidal waves applied to electrode array.

i.e., the complex potential is $\Phi=\sum_{p=0}^{\infty} \Phi_{p} \mathrm{e}^{i(2 p+1) \omega t}$. It can be seen that the potentials $\Phi_{p}$ with $p=$ even are traveling waves going to the right in Fig. 2 while the potentials with $p$ =odd go to the left. The time-averaged slip velocity generated is

$$
u=-\frac{\varepsilon}{\eta}\left\langle\Delta \Phi \frac{\partial \Phi}{\partial x}\right\rangle=-\frac{\varepsilon}{2 \eta} \sum_{p=0}^{\infty} \operatorname{Re}\left[\Delta \Phi_{p} \frac{\partial \Phi_{p}^{*}}{\partial x}\right] .
$$

Each term of the series can be computed using the numerical scheme of the present section, taking into account the corresponding frequency $(2 p+1) \omega$, voltage amplitude $4 V_{0} / \pi(2 p$ +1 ), and direction of the wave, i.e., $u=\sum_{p=0}^{\infty}(-1)^{p} u_{p}$.

\section{NUMERICAL RESULTS AND DISCUSSION AT LOW VOLTAGE}

The numerical convergence was verified through multiple runs of increasing number of functions. The estimated error is less than $0.1 \%$ using $n=64$ functions. For the case $d_{1}=d_{2}=d$, the maximum pumping velocity as a function of frequency is $U=0.0502 \Lambda \varepsilon k_{0} V_{0}^{2} / \eta$ obtained at a frequency $\omega=1.4492 \sigma k_{0} / C_{\mathrm{DL}}$. A plot of the pumping velocity (in units of $\Lambda \varepsilon k_{0} V_{0}^{2} / 4 \eta$ ) as a function of frequency (in units of $\left.\sigma k_{0} / C_{\mathrm{DL}}\right)$ is shown in Fig. 8. For comparison, the pumping velocity obtained from a pure traveling wave, Eq. (10), and from a traveling square wave applied to the electrode array is also presented. The computations show that the maximum slip velocity in a four-phase microelectrode array with $d_{1}$ $=d_{2}$ is around five times smaller than the maximum velocity obtained by a pure potential traveling wave of voltage amplitude $V_{0}$ and wave number $k_{0}=2 \pi / L$. The main reason for this difference is that the slip velocity is zero on the gaps between electrodes, the resulting pumping velocity being the average over a wavelength.

The pumping velocity generated by a four-phase square wave of maximum voltage $V_{0}$ shows a maximum pumping velocity as a function of frequency of $U=0.0762 \Lambda \varepsilon k_{0} V_{0}^{2} / \eta$. If only the first harmonic of the Fourier series in $\omega$ is employed [curve (c) in Fig. 8 multiplied by $16 / \pi^{2}$ ], the maximum value is around $7 \%$ greater than the one obtained using the complete Fourier series.

The streamlines generated by the slip velocity, Eq. (7), are shown in Fig. 9 for two frequencies: $\Omega=\Omega_{M} / 5$ and $\Omega$ $=5 \Omega_{M}$, with $\Omega_{M}=1.45$ the nondimensional frequency of

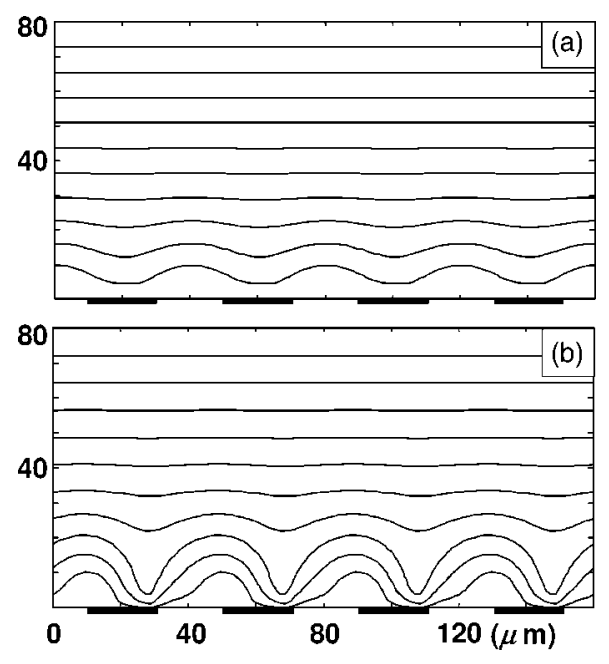

FIG. 9. Streamlines for the cases: (a) $\Omega=\Omega_{M} / 5$, (b) $\Omega=5 \Omega_{M}$. Here $\Omega_{M}$ $=1.45$, the nondimensional frequency of maximum velocity.

maximum pumping velocity. In this figure, the traveling wave goes from right to left in order to compare with experimental streamlines. As the frequency increases the slip velocity is less uniform on top of each electrode. The experimental streamlines show a qualitative agreement with the computed ones.

These computed streamlines can be compared with those obtained numerically ${ }^{15}$ and experimentally ${ }^{32}$ for asymmetric pairs of electrodes. The fluid flow for TWEO is more uniform, and this can be an advantage in circular chromatographic applications. ${ }^{33}$ We can also compare the theoretical slip velocity for TWEO and for ac electroosmosis in asymmetric electrodes in a representative case. The predicted pumping velocity for the asymmetric array of Brown et al. ${ }^{8}$ is given in Ref. $15, U=0.52 \Lambda V_{0}^{2} \mathrm{~mm} / \mathrm{s}$. Here $V_{0}$ is the amplitude of the ac voltage applied to each electrode, i.e., the maximum potential difference between electrodes is $2 V_{0}$. The theoretical velocity for a TWEO experiment with an array of the same wavelength $(L=50 \mu \mathrm{m})$ is $U$ $=4.5 \Lambda V_{0}^{2} \mathrm{~mm} / \mathrm{s}$. The TWEO velocity is a factor of the order of 10 greater for the same voltage amplitude and, in conclusion, it suggests that the TWEO is more efficient.

The predicted frequency for maximum velocity in our experiments is $f=776 / \Lambda \mathrm{Hz}$. A value of $\Lambda \sim 0.4$ is needed in order to obtain the experimental value around $2 \mathrm{kHz}$. The existence of a layer of oxide that naturally forms on top of the titanium can account for this factor. Also, in Ref. 13 we had a better agreement with experiments if we used a measured double-layer impedance in boundary condition (2), rather than the impedance given by perfect capacitors, i.e., $1 /\left(i \omega C_{\mathrm{DL}}\right)$.

It is clear that the experimental velocities presented in Fig. 5 do not show a quadratic dependence on voltage. This is not completely unexpected since the Debye-Huckel approximation of the double layer (linear theory) can only be used tentatively for voltages across the diffuse layer greater than the thermal voltage $\left(k_{B} T / e=0.025 \mathrm{~V}\right)$. For our system, the linear theory predicts a maximum pumping velocity $U$ $=\Lambda 1.38 V_{0}^{2} \mathrm{~mm} / \mathrm{s}\left(V_{0}\right.$ in volts $)$. A factor $\Lambda \sim 0.05$ is needed to fit the experimental velocities for low voltages $\left(V_{0}\right.$ 
$\sim 0.5 \mathrm{~V}$ ). The oxide layer on top of the titanium electrode can account in part for the reduction of the experimental velocities. Other factors that can reduce the maximum achievable velocity are the three-dimensional nature of the flow, together with the front window that will increase the viscous friction.

The traveling wave experiments presented in Ref. 17 show a frequency of maximum velocity of the order of $\sigma / \varepsilon$ because of the existence of an insulating layer between the electrodes and the electrolyte. Although those results cannot be analyzed with the present theory, valid for $\omega \varepsilon / \sigma \ll 1$, we can try to draw some conclusions. Cahill et al. obtained a quantitative difference between their predicted and experimental velocities. They simulated the imposed voltage as a pure sinusoidal traveling wave. When we take into account the actual geometry of our electrode array (very thin electrodes separated by gaps, mounted on an insulating glass substrate), the calculated velocity is around five times smaller than the one expected from a single mode of frequency $\omega$, wave number $k$, and voltage amplitude $V_{0}$. Our results indicate that a quantitative comparison between theoretical and experimental slip velocities should take into account the actual geometry and material properties of the system. We would expect the theoretical velocities calculated by Cahill et al. to be smaller when the actual shape of the electrodes is included in the computations. With respect to the slip velocity distribution, we would expect that this is much smaller on the gaps than on the electrodes.

\section{CONCLUSIONS}

Net flow of electrolyte induced by a traveling-wave potential applied to an array of microelectrodes has been reported. The observed flows seem to be driven at the level of the electrodes. The results have been analyzed with a linear electroosmotic model based upon the Debye-Huckel theory. The electrical problem for the experimental interdigitated electrodes was solved numerically using a truncated Fourier series. The linear analysis shows qualitative accordance with the observations at low voltages. At applied voltage amplitudes greater than $2 \mathrm{~V}$ and at frequency of $1 \mathrm{kHz}$, the fluid flow changed direction. By increasing the voltage, this reversal flow can easily be ten times faster than the one at low voltages. More experimental and theoretical research is needed to clarify the origin of this kind of flow. The possibility of switching the fluid flow direction with increasing voltage may be of interest in the lab-on-a-chip technology. ${ }^{25}$

We have analyzed a problem with $L \ll h \ll w$, where $h$ is the height of the upper wall and $w$ the depth of the array. In this situation, since the electroosmotic slip velocity is $U$, the maximum flow rate that can be generated by the array of electrodes is $F=U h w / 2$, taking into account that the upper wall boundary condition is of nonslip. The pressure drop that can be generated in a unit wavelength is given by $p_{b}$ $=12 \eta L F / w h^{3}=6 \eta U L / h^{2}$. For the experimental array and a slip velocity of $50 \mu \mathrm{m} / \mathrm{s}$, this gives $p_{b} \sim 10^{-3} \mathrm{~Pa}$ per wavelength. In order to increase the pressure drop, the number of cells should be increased but even to generate $1 \mathrm{~Pa}$ would require a pump with 1000 unit cells; $160 \mathrm{~mm}$ for this design of pump. Clearly, the height of a device and the wavelength could be reduced in order to increase the pressure drop. However, these kinds of pumps have many potential applications for manipulating particles and fluids in closed microdevices, which do not require high pressure. ${ }^{33}$

\section{ACKNOWLEDGEMENT}

The authors would like to thank the Spanish government agency Dirección General de Ciencia y Tecnología under Contract No. BFM2003-01739.

${ }^{1}$ G. M. Whitesides and A. Stroock, Phys. Today 54(6), 42 (2001).

${ }^{2}$ H. A. Stone, A. D. Stroock, and A. Ajdari, Annu. Rev. Fluid Mech. 36, 381 (2004).

${ }^{3}$ H. T. G. van Lintel, Sens. Actuators 15, 153 (1988).

${ }^{4}$ A. Manz, C. S. Effenhauser, N. Burggraf , D. J. Harrison, K. Seiler and K. Fluri, J. Micromech. Microeng. 4, 257 (1994).

${ }^{5}$ G. Beni and M. A. Tenan, J. Appl. Phys. 52, 6011 (1995).

${ }^{6}$ D. E. Kataoka and S. M. Troian, Nature (London) 402, 794 (1999).

${ }^{7}$ G. Fuhr, R. Hagedorn, T. Müller, W. Benecke, and B. Wagner, J. Microelectromech. Syst. 1141 (1992).

${ }^{8}$ A. B. D. Brown, C. G. Smith, and A. R. Rennie, Phys. Rev. E 63, 016305 (2000).

${ }^{9}$ A. Ramos, H. Morgan, N. G. Green, and A. Castellanos, J. Colloid Interface Sci. 217, 420 (1999).

${ }^{10}$ A. Ajdari, Phys. Rev. E 61, R45 (2000).

${ }^{11}$ N. G. Green, A. Ramos, A. González, H. Morgan, and A. Castellanos, Phys. Rev. E 61, 4011 (2000).

${ }^{12}$ A. González, A. Ramos, N. G. Green, A. Castellanos, and H. Morgan, Phys. Rev. E 61, 4019 (2000).

${ }^{13}$ N. G. Green, A. Ramos, A. González , H. Morgan, and A. Castellanos, Phys. Rev. E 66, 026305 (2002).

${ }^{14}$ M. Z. Bazant and T. M. Squires, Phys. Rev. Lett. 92, 066101 (2004)

${ }^{15}$ A. Ramos, A. González, A. Castellanos, N. G. Green, and H. Morgan, Phys. Rev. E 67, 056302 (2003).

${ }^{16}$ R. M. Ehrlich and J. R. Melcher, Phys. Fluids 25, 1785 (1982).

${ }^{17}$ B. P. Cahill, L. J. Heyderman, J. Gobrecht, and A. Stemmer, Phys. Rev. E 70, 036305 (2004).

${ }^{18}$ A. Ramos, H. Morgan, N. G. Green, and A. Castellanos, J. Phys. D 31, 2388 (1998).

${ }^{19}$ A. Castellanos, A. Ramos, A. González, N. G. Green, and H. Morgan, J. Phys. D 36, 2584 (2003).

${ }^{20}$ Y. Huang, X. B. Wang, J. A. Tame, and R. Pethig, J. Phys. D 26, 1528 (1993).

${ }^{21}$ H. Morgan, A. García, D. Bakewell, N. G. Green, and A. Ramos, J. Phys. D 34, 1553 (2001).

${ }^{22}$ N. G. Green, A. Ramos, and H. Morgan, J. Electrost. 56, 235 (2002).

${ }^{23}$ L. Cui, D. Holmes, and H. Morgan, Electrophoresis 22, 3893 (2001).

${ }^{24}$ Experiments reported by N. G. Green at Nanotech 2003 in Montreaux, Switzerland.

${ }^{25}$ V. Studer, A. Pepin, Y. Chen, and A. Ajdari, Analyst (Cambridge, U.K.), 129, 944 (2004).

${ }^{26}$ M. Z. Bazant, K. Thornton, and A. Ajdari, Phys. Rev. E 70, 021506 (2004).

${ }^{27}$ J. L. Anderson, Annu. Rev. Fluid Mech. 21, 61 (1989).

${ }^{28}$ A. P. Washabaugh, M. Zahn, and J. R. Melcher, IEEE Trans. Electr. Insul. 24, 807 (1989).

${ }^{29}$ D. Lastochkin, R. Zhou, P. Wang, Y. Ben, and H. C. Chang, J. Appl. Phys. 96, 1730 (2004).

${ }^{30}$ J. Gunning, D. Y. C. Chan, and L. R. White, J. Colloid Interface Sci. 170 522 (1995).

${ }^{31}$ V. G. Levich, Physicochemical Hydrodynamics (Prentice-Hall, Englewood Cliffs, NJ, 1962).

${ }^{32}$ A. Ramos, A. González, A. Castellanos, N. G. Green, and H. Morgan, Inst. Phys. Conf. Ser. 178, 187 (2004).

${ }^{33}$ S. Debesset, C. J. Hayden, C. Dalton, J. C. T. Eijkel, and A. Manz, Lab Chip 4, 396 (2004). 